\title{
Concezione e realizzazione del Questionario per la valutazione della didattica e della relazione con l'insegnante
}

\section{Giorgio Ostinelli}

La valutazione dell'insegnamento è sempre stata ed è tuttora un tema allo stesso tempo importante e di difficile attuazione. Benché chi insegna valuti in continuazione, si constata tuttavia che, nella maggior parte dei casi, lelgli insegnanti non amano essere valutati. Come fare per introdurre delle forme di valutazione "sostenibili», che aiutino a meglio mettere a fuoco aspetti importanti dell'insegnamento e che siano allo stesso tempo rigorose e rispettose della professionalità $e$ della personalità delleldei docenti?

La creazione di un contesto orientato al miglioramento nelle scuole può essere un'occasione importante per sviluppare approcci dotati di tali caratteristiche, ed ̀̀, in poche parole, quanto è avvenuto presso la Scuola professionale e artigianale (SPAI) di Mendrisio negli ultimi anni. Nei fatti, nell'ambito del progetto DAAPI, sorto a partire da un'iniziativa federale volta a introdurre forme di autovalutazione negli istituti scolatici, un gruppo di lavoro a cui prendevano parte rappresentanti delle varie categorie di attori presenti nella scuola (insegnanti, allievi, direzione, ecc.) ha sviluppato, con l'assistenza di uno School Improvement Advisor/researcher (SIA), un questionario per la valutazione della didattica e della relazione con l'insegnante. Il presente articolo descrive le tappe che hanno condotto alla creazione di tale strumento e le procedure che hanno permesso di affinarne la validità e l'affidabilità, nell'ottica di una sua utilizzazione su più larga scala da parte dei docenti ticinesi e di madrelingua italiana.

\section{Perché valutare l'insegnamento?}

Valutare alcuni aspetti attinenti alle pratiche di insegnamento messe in atto dai docenti riveste una grande importanza in quanto permette sia all'insegnante stesso, sia all'istituto scolastico di disporre di informazioni utili a caratterizzare tanto lo status quo che le possibilità di evoluzione della situazione presa in esame. In particolare questo secondo fattore risulta oggi di grande importanza, in un momento in cui la scuola è toccata da cambiamenti che avvengono in tempi nettamente più rapidi rispetto ad un passato anche recente. 
La valutazione dell'insegnamento è quindi una tematica di notevole importanza (Ellett \& Teddlie, 2007; Peterson, 2000a, 2000b). Tuttavia, a dispetto di questo fatto, è noto come essa abbia suscitato e continui a suscitare un certo numero di perplessità, in particolare fra gli insegnanti, che sospettano che dietro alle buone intenzioni «di facciata» si celi in realtà una volontà di limitare la loro libertà d'azione: le loro reticenze possono probabilmente essere spiegate dal fatto che chi passa una buona parte del proprio tempo a valutare gli altri non desidera, per vari motivi, essere a sua volta oggetto di valutazione (Stumpf \& Rindova, 1997).

Il presente articolo si pone due scopi principali: da un lato produrre una riflessione sulla pratica professionale degli insegnanti e sulle relative modalità di cambiamento, e dall'altro proporre un questionario rivolto agli allievi e finalizzato alla valutazione dell'insegnamento, del quale vengono contemporaneamente illustrate le procedure volte ad una sua valutazione.

\section{Letteratura sull'argomento}

Realizzare delle valutazioni che abbiano quale oggetto la prestazione professionale degli insegnanti è un tema di notevole complessità. Al di là dell'apparente semplicità dell'interazione tra docente ed allievi, vi sono vari fattori di tipo socioculturale, ambientale, generazionale, ecc., che esercitano la loro influenza. Darling-Hammond, Wise e Pease (1983) sottolineano come le aspettative in materia varino sensibilmente tra categorie interessate, quali insegnanti, dirigenti, genitori; oltre a ciò, la differenza funzionale e operativa esistente tra approcci formativi e sommativi non è sempre percepita in modo chiaro dai soggetti che prendono parte al processo (Scriven, 1987). Malgrado alcuni progressi realizzati nel corso degli anni, la valutazione dell'insegnamento è tuttora praticata mediante modalità non sempre rigorose (Peterson, 2000a). Indipendentemente della correttezza metodologica dei vari interventi, questi possono essere suddivisi grosso modo in due filoni: quello che si pone come obiettivo l'assicurazione della prestazione di qualità del sistema, che prevede in genere solitamente valutazioni esterne di tipo standardizzato, e quello che concepisce invece la valutazione come un mezzo per promuovere il miglioramento della professionalità degli insegnanti, facendo leva piuttosto su forme di autovalutazione e di riflessione. Un volume come The Personnel Evaluation Standards (Joint Committee on Standards for Educational Evaluation - JCSEE, 1988) può essere preso come esempio del primo tipo di approccio, mentre un'opera come Valutare l'insegnamento (Sergiovanni \& Starratt, 2003) rappresenta bene la seconda tendenza. In lingua italiana, per quanto riguarda il tema trattato in questa sede, al di là dei volumi generali in materia di valutazione (Vertecchi, 2003), oppure sulla valutazione degli istituti scolastici (Bottani \& Cenerini, 2003), esistono sia opere tradotte dall'inglese (OCSE/CERI, 1994), sia articoli pubblicati su riviste da autori italiani. Castoldi, ad esempio, sottolinea come il docente dovrebbe essere 
concepito come professionista riflessivo operante all'interno di un contesto. Sulla base di questa visione, propone un modello sistemico nel quale aspetti formativi e criteri di valutazione si completano reciprocamente (Castoldi, 1999, 2004). Ribolzi (2011) dal canto suo, nella sua panoramica sul sistema scolastico italiano e sul suo contrastato rapporto con il tema della valutazione degli insegnanti, pur ponendo l'accento sulla necessità di disporre di standard di sistema, sottolinea l'importanza del miglioramento della prestazione professionale dei docenti e il ruolo chiave dei dirigenti scolastici in tale contesto. Infine Comoglio (2011) propone di superare l'antinomia tra ragioni a favore e ragioni contro la valutazione dell'insegnamento, considerando queste ultime come condizioni delimitanti. L'approccio proposto è centrato principalmente sul miglioramento della prestazione professionale dei docenti.

Passando ora più nel dettaglio alla valutazione dell'insegnamento mediante questionari destinati agli allievi, esiste una letteratura di una certa consistenza in materia (Aleamoni, 1981; Angelo \& Cross, 1993; Kulik, 2001; Stevens, 1987; Svinicki, 2001; Theall \& Franklin, 2001). Gli autori citati mettono in evidenza come i giudizi espressi dagli studenti si rivelino in genere validi, nella misura in cui le questioni loro sottoposte facciano parte del loro dominio di competenza; l'affidabilità è pure comprovata, sia entro che fra classi scolastiche, oppure sull'arco del tempo (Marsh, 1987). Un fatto che viene sottolineato da più parti è come la pura e semplice comunicazione dei risultati agli insegnanti non produca solitamente effetti degni di rilievo, i quali si verificano invece quando i dati vengono a far parte di un processo di consulenza rivolto al docente (Aleamoni, 1981; McKeachie, 1987; Stevens, 1987).

\section{La valutazione degli insegnanti nel quadro dell'autovalutazione globale di un istituto scolastico}

L'approccio alla valutazione dell'insegnamento che verrà descritto nelle prossime pagine è stato sviluppato presso la Scuola professionale artigianale e industriale (SPAI) di Mendrisio, nell'ambito di un vasto progetto relativo all'autovalutazione di un gruppo di istituti scolastici svizzeri del settore professionale - il progetto DAAP - concepito e messo in atto con la supervisione di John MacBeath, noto esperto internazionale di School Improvement (Berger et al., 2004). La SPAI di Mendrisio è una scuola professionale frequentata da circa 850 apprendisti, con un corpo docente costituito da 30 insegnanti. Essa offre una formazione nell'ambito dell'edilizia, dell'elettricità e della macelleria.

La procedura di autovalutazione, mediante la partecipazione degli stakeholders della scuola (insegnanti, allievi, rappresentanti della direzione, datori di lavoro) a un gruppo operativo (GO), ha condotto all'identificazione di un certo numero di aspetti da migliorare, tra cui vi erano la didattica e le relazioni fra docenti e 
allievi. Tale corso di azione è stato seguito e messo a punto in modo efficace grazie alla partecipazione di uno School Improvement Advisor / researcher (SIA), una figura professionale che svolge un ruolo per così dire "catalitico", e che accomuna in sé le caratteristiche del critical friend con quelle del ricercatore e con quelle del consulente educativo: nei fatti, egli contribuisce a far sì che gli istituti scolastici mettano in atto procedimenti di ricerca-azione sul campo, contraddistinti da adeguati criteri di rigore e di solidità (Ostinelli, 2007, 2008a, 2008b, 2009).

\section{La costruzione condivisa di uno strumento di valutazione}

In questo contesto, il GO ha elaborato, con la consulenza del SIA, un questionario destinato agli allievi, con l'obiettivo di valutare la didattica e la relazione fra insegnanti e allievi. Questi due aspetti sono stati scelti perché permettono agli allievi di formulare giudizi con competenza: non avrebbe, infatti, molto senso chiedere loro pareri su altri aspetti caratteristici dell'attività svolta dall'insegnante, quali ad esempio la sua preparazione disciplinare. Benché vi siano diverse tecniche per valutare l'insegnamento, come ad esempio, tanto per citarne alcune, i portfolio e le visite di pari (Peterson, 2000a), la scelta del GO è caduta sulla valutazione tramite questionario. A questo proposito, è importante ribadire come la pura e semplice utilizzazione di questo genere di strumenti non sia sufficiente per ottenere spontaneamente dei miglioramenti dagli insegnanti (McKeachie, 1987): nei fatti, presso la SPAI di Mendrisio il questionario è stato parte di una più ampia azione di consulenza a livello istituzionale e individuale messa in atto dal SIA. Occorre infine sottolineare come vi sia una grande differenza, in termini motivazionali, tra forme di valutazione scelte, progettate e negoziate all'interno della scuola e modalità analoghe decise e prescritte da autorità esterne, ossia quando si gioca il ruolo di «pedina» oppure di «origine» (De Charms, 1968; Deci \& Ryan, 1985; Ostinelli, 2005).

Il GO è stato in primo luogo invitato a definire un certo numero di item rispetto ai quali gli allievi avessero le necessarie competenze per poter esprimere delle valutazioni. Il gruppo ha così proposto delle possibili questioni che sono poi state riformulate con l'assistenza del SIA, con la finalità di farne dei veri e propri item adatti per la somministrazione tramite questionario. A questi ne sono stati infine aggiunti altri, scelti dal GO da una lista realizzata a partire da strumenti simili comunemente usati, in particolare nel mondo anglosassone (Lewis, 2001; Peterson, 2000a; Wilson, 1987). La procedura qui descritta ha condotto il GO a definire - e il SIA a realizzare tecnicamente - un questionario comprendente 32 item e una scala di frequenza a cinque posizioni (mai/raramente/ogni tanto/ spesso/sempre). Si è poi proceduto a una prima somministrazione sperimentale, sotto forma di pre-test, in due classi, con il coinvolgimento volontario di due insegnanti: questo fatto si è tra l'altro rivelato di grande utilità nel convincere 
i colleghi a partecipare alla somministrazione definitiva, avvenuta nel corso dell'anno scolastico 2004-2005.

Seguendo le indicazioni dei reviewer, è opportuno presentare qui la struttura del questionario (dimensioni principali, sezioni delle domande), e presentare un paio di item come esempio.

\section{L'utilizzazione dello strumento}

L'elaborazione dei risultati è avvenuta a cura della scuola, sotto la supervisione del SIA, e gli esiti sono stati poi comunicati agli insegnanti; il SIA ha quindi preso contatto con i docenti operanti almeno a metà tempo, proponendo un colloquio su base volontaria, accolto favorevolmente dal 75\% di essi $(100 \%$ nella materia cultura generale). I colloqui hanno avuto durata variabile, da circa una a cinque-sei ore, come nel caso che verrà illustrato più avanti nel testo, a dipendenza delle carenze messe in evidenza dallo strumento. Una seconda somministrazione è stata poi effettuata durante l'anno scolastico 2006-2007, e gli insegnanti che hanno preso parte anche a questa seconda somministrazione hanno potuto confrontare i dati relativi ai due momenti. Il SIA ha contestualmente sviluppato un'analisi dei risultati e, sulla base della significatività statistica (test $\mathrm{t}$ ), ha messo in luce gli aspetti che hanno subito la maggiore variazione in una serie di rapporti individualizzati, che sono serviti come base per un ulteriore colloquio con i docenti: in molti casi il cambiamento rifletteva degli interventi intenzionalmente messi in atto dagli insegnanti stessi, come illustrato nella figura seguente, che riporta in sintesi la reazione di un docente ad una lacuna segnalata dagli allievi:

19. Gli strumenti (computer, lucidi, ecc.) utilizzati dal docente mi aiutano a meglio comprendere la lezione

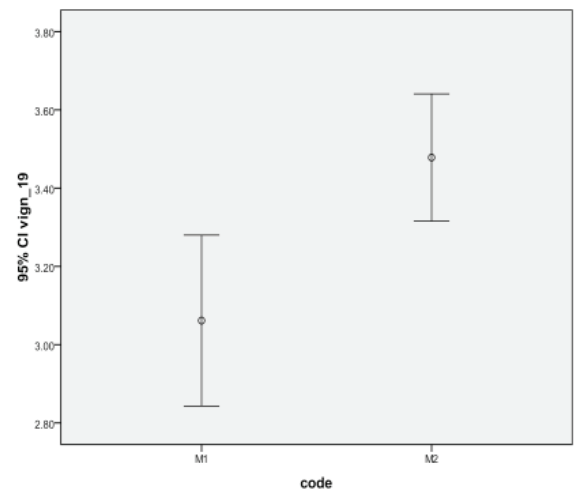

Figure 1: Item 19. I dati mostrano un miglioramento facente seguito a cambiamenti introdotti dall'insegnante. $M 1(2004)=3.06 M 2(2007)=3.47 ; t(109)=2.84, p<0.005$ 
Il test si è pure rivelato particolarmente utile al momento in cui è stata sperimentata l'introduzione di innovazioni di un certo peso a livello didattico in alcune classi, in particolare con l'introduzione della metodologia Understanding by Design (Wiggins \& McTighe, 1998), consentendo un raffronto con altre classi che non avevano partecipato all'esperienza (Ostinelli, 2008b)

Dopo la somministrazione del questionario, il fatto di aver realizzato dei colloqui con gli insegnanti, destinati a mettere in atto un dialogo costruttivo, si è rivelato un fattore di grande importanza nell'approfondimento e nell'interpretazione dei dati rilevati mediante il questionario stesso. Ad esempio, nel caso di un secondo docente, un risultato globalmente insoddisfacente ha mostrato, a un'analisi più attenta, di aver origine esclusivamente in una classe, in cui sussisteva un conflitto tra il docente e un gruppo di allievi. Di conseguenza, la riflessione si è focalizzata sui rapporti e sulle conflittualità specifiche, piuttosto che su lacune generalizzate nell'approccio del docente, ponendosi l'obiettivo di agire preventivamente se si fossero venuti a manifestare casi analoghi in futuro. Le due figure seguenti illustrano il caso in questione:

30. L'insegnante agisce in modo equo

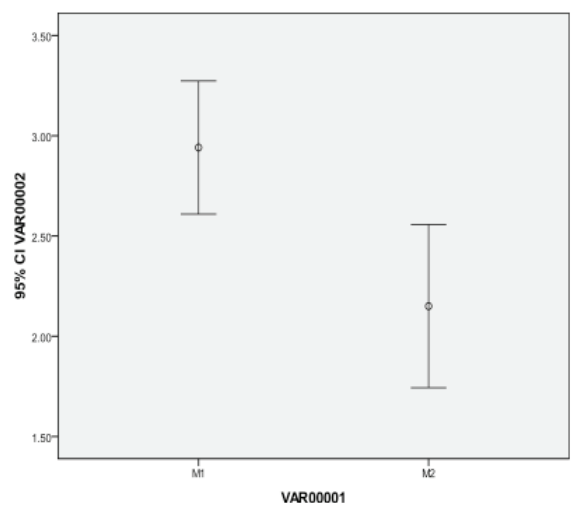

Figure $2:$ Item 30 con tutte le classi. $M 1(2004)=2.94 M 2(2007)=2.15 ; t$ $(72)=2.99, p<0.004:$ la differenza tra le medie è statisticamente significativa e indica un peggioramento

La constatazione che sorge da quanto illustrato in questo caso è che il questionario ha espresso il suo valore effettivo nel quadro di un'azione indirizzata al miglioramento, debitamente concepita e pianificata in collaborazione con il SIA. Diversamente, gli effetti sarebbero stati marginali - al di là dei fraintendimenti che una lettura superficiale del dato può comportare - come spesso avviene quando lo scopo si limita alla mera comunicazione dei risultati alle persone o alle istanze dirigenti (Aleamoni, 1981; McKeachie, 1987). 
30. L'insegnante agisce in modo equo

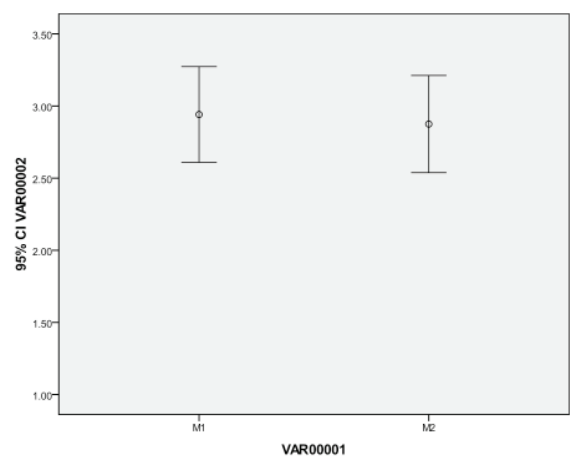

Figure 3 : Item 30 senza la classe problematica. $M 1(2004)=2.94$ M2 (2007)= 2.88; $t(56)=0.28, p<0.78$ : la differenza non è statisticamente significativa. Il problema è quindi "localizzato" in una sola classe.

È importante sottolineare come l'esperienza condotta ci abbia portato a concludere che la scelta e l'uso degli strumenti, delle modalità di valutazione da mettere in campo e delle loro caratteristiche di realizzazione, avvengano in modo ideale all'interno di un piano più vasto, basato su una filosofia e degli obiettivi di fondo, che, nel caso in questione erano rappresentati dal progetto di autovalutazione di istituto, mirante a costituire una comunità che apprende. Di conseguenza, la valutazione messa in atto ha avuto un carattere essenzialmente formativo, destinato a indurre gli insegnanti a riflettere sulle loro pratiche didattiche e sulle loro maniere di proporsi nei confronti degli allievi, ponendosi l'obiettivo di migliorare il loro insegnamento e di sviluppare delle opportune strategie preventive.

\section{Quadro scientifico e metodologia}

Il Questionario per la valutazione della didattica e della relazione con l'insegnante si pone come strumento utile a migliorare la prestazione professionale dell'insegnante nell'ottica di una sua riflessione assistita sulle proprie pratiche di insegnamento. Tale concezione ottempera a quanto emerso dalla ricerca scientifica in materia (Aleamoni, 1981; McKeachie, 1987) e si situa nell'ottica dello sviluppo di una professionalità riflessiva, secondo una modalità auspicata anche da vari autori (Castoldi, 2004; Schön, 1987; Sergiovanni \& Starratt, 2003). In effetti, come sottolineato anche in altre sedi (Ostinelli, 2007, 2008a, 2008b), è possibile sviluppare in ambito scolastico interventi a livello "micro», in particolare in tema di miglioramento delle pratiche professionali di insegnamento, che abbinino un adeguato standard di rigore a un approccio di tipo «ecologico», ossia rispettoso, tra le altre cose, della professionalità docente. 
In quest'ottica, la comprovata utilità a livello empirico dello strumento presentato in queste pagine ha spinto il SIA a prenderne in considerazione una possibile validazione, anche per poterne estendere l'uso ad altri istituti scolastici di lingua italiana. Di conseguenza, il questionario è stato sottoposto a una verifica dell'affidabilità, mediante test $\alpha$ di Cronbach, mentre, per verificarne la validità è stata condotta un'analisi fattoriale esplorativa, che ha condotto, come in genere avviene in questi casi, a una riduzione del numero degli item. Questi ultimi sono stati in un secondo tempo sottoposti a un panel di 15 giudici (11 docenti e 4 ricercatori operanti nel contesto scolastico ticinese, scelti in base alla loro disponibilità) per una validazione di contenuto. In particolare, i giudici avevano il compito di classificare gli item in base alle tre principali dimensioni emerse dall'analisi. Per realizzare il procedimento qui descritto sono stati analizzati i risultati relativi a due somministrazioni, quella del 2004 e quella del 2007.

\section{Analisi statistica dell'affidabilità e della validità del Questionario per la valutazione della didattica e della relazione con l'inse- gnante}

Come detto, lo strumento è stato innanzitutto sottoposto al test $\alpha$ di Cronbach, per verificare il valore dell'omonimo coefficiente. Per la somministrazione del 2004 (278 soggetti), il risultato è stato di 0.92, mentre per quella del 2007 (301 soggetti) è stato di 0.94 . Di conseguenza, si può affermare che il questionario gode di un livello di affidabilità elevato, che si mantiene nel tempo.

L'analisi fattoriale esplorativa in base al metodo delle componenti principali, condotta su tutti i 32 item del test, ha evidenziato 7 possibili fattori; tuttavia, tenendo in conto il fatto che i primi 3 spiegano il 43\% (48\% nel 2007) della varianza e i rimanenti 4 solo circa il $15 \%$ (10\% nel 2007), per motivi di semplicità si è ridotto il modello a tre fattori.

Tableau 1: pesi dei fattori e varianza spiegata, 2004

\begin{tabular}{|c|c|c|c|}
\hline \multicolumn{2}{|c|}{ Pesi dei fattori non ruotati } & \multicolumn{2}{c|}{ Pesi dei fattori ruotati } \\
\hline \% di varianza & \% cumulata & \% di varianza & \% cumulata \\
\hline 32.419 & 32.419 & 18.783 & 18.783 \\
\hline 6.356 & 38.775 & 18.367 & 37.150 \\
\hline 4.481 & 43.256 & 6.106 & 43.256 \\
\hline
\end{tabular}


Tableau 2: pesi dei fattori e varianza spiegata, 2007

\begin{tabular}{|c|c|c|c|}
\hline \multicolumn{2}{|c|}{ Pesi dei fattori non ruotati } & \multicolumn{2}{c|}{ Pesi dei fattori ruotati } \\
\hline \% di varianza & \% cumulata & \% di varianza & \% cumulata \\
\hline 38.173 & 38.173 & 25.818 & 25.818 \\
\hline 5.973 & 44.146 & 17.069 & 42.887 \\
\hline 4.355 & 48.501 & 5.613 & 48.501 \\
\hline
\end{tabular}

I dati, per quanto riguarda la varianza totale spiegata, mostrano come il questionario ottenga dei buoni risultati, avvicinandosi circa al 50\%. L'applicazione di una rotazione Varimax conduce inoltre ad una più conveniente distribuzione dei pesi tra i fattori, individuando in modo abbastanza netto tre possibili dimensioni. Passando ora a un'analisi più dettagliata del peso dei singoli item e della loro dimensionalità, ecco i risultati:

Tableau 3: matrice dei componenti dei tre fattori presi in esame

\begin{tabular}{|l|r|r|r|r|r|r|r|}
\hline & \multicolumn{3}{|c|}{ Componenti 2003} & \multicolumn{5}{c|}{ Componenti 2007} \\
\cline { 2 - 8 } & 1 & 2 & 3 & & 1 & 2 & \multicolumn{1}{c|}{3} \\
\hline VAR00004 & .664 & .234 &. .105 & VAR00030 & .849 & .197 & .063 \\
\hline VAR00005 & .655 & .148 & .001 & VAR00031 & .792 & .179 & -.086 \\
\hline VAR00013 & .634 & .380 & .109 & VAR00025 & .731 & .228 & .090 \\
\hline VAR00001 & .620 & .097 & .160 & VAR00017 & .731 & .125 & .028 \\
\hline VAR00022 & .605 & .376 & .121 & VAR00027 & .701 & .347 & .015 \\
\hline VAR00002 & .590 &. .029 & .344 & VAR00009 & .688 & .349 & .032 \\
\hline VAR00021 & .581 & .118 & .299 & VAR00016 & .683 & .256 & .235 \\
\hline VAR00003 & .570 & .125 & .101 & VAR00015 & .677 & .355 & .062 \\
\hline VAR00027 & .508 & .477 & .146 & VAR00028 & .623 & .275 &. .037 \\
\hline VAR00008 & .489 & .274 & .132 & VAR00020 & .611 & .166 & -.151 \\
\hline VAR00009 & .436 & .434 & .012 & VAR00022 & .566 & .453 & .046 \\
\hline VAR00006 & .431 & .375 &. .195 & VAR00018 & .546 & .440 & .083 \\
\hline VAR00024 & .415 & .337 & .286 & VAR00024 & .535 & .410 & .001 \\
\hline VAR00019 & .376 & .167 & .160 & VAR00029 & .532 & .408 & .188 \\
\hline VAR00015 & .140 & .779 & -.013 & VAR00012 & .530 & .457 & .171 \\
\hline VAR00016 & .094 & .760 & .091 & VAR00013 & .455 & .452 & .260 \\
\hline VAR00017 & .064 & .660 & .082 & VAR00014 & .442 & .382 & .174 \\
\hline VAR00025 & .424 & .644 & .052 & VAR00023 & .390 & .358 & -.006 \\
\hline VAR00030 & .423 & .613 & .021 & VAR00004 & .197 & .645 & .126 \\
\hline
\end{tabular}




\begin{tabular}{|l|r|r|r|l|r|r|r|}
\hline VAR00018 & .333 & .560 & .122 & VAR00003 & .227 & .642 & -.118 \\
\hline VAR00028 & .418 & .556 & .113 & VAR00005 & .229 & .635 & -.006 \\
\hline VAR00029 & .270 & .541 & .248 & VAR00002 & .298 & .598 & -.195 \\
\hline VAR00012 & .524 & .532 & .103 & VAR00001 & .315 & .556 & -.053 \\
\hline VAR00031 & .461 & .492 & .092 & VAR00021 & .304 & .541 & -.092 \\
\hline VAR00026 & .009 & .446 & .424 & VAR00011 & .384 & .455 & -.097 \\
\hline VAR00014 & .338 & .406 & .064 & VAR00008 & .441 & .450 & .131 \\
\hline VAR00011 & .109 & .391 & .264 & VAR00026 & .347 & .431 & -.047 \\
\hline VAR00007 & .193 & -.231 & .575 & VAR00010 & .014 & .386 & -.328 \\
\hline VAR00010 & -.157 & .244 & .536 & VAR00006 & .075 & .021 & .724 \\
\hline VAR00023 & .374 & .115 & .507 & VAR00007 & .033 & .251 & -.711 \\
\hline VAR00020 & .195 & .152 & .415 & VAR00019 & .145 & .444 & .468 \\
\hline
\end{tabular}

Gli item appartenenti ai tre fattori oggetto dell'analisi sono evidenziati in tre diverse gradazioni (grigio scuro, grigio chiaro, bianco. I primi due, che nel 2004 avevano un peso complessivo praticamente equivalente, nella tabella del 2007 si sono scambiati di posto, dal momento che il secondo ha assunto un peso leggermente maggiore, come appare dalla tabella.

Analizzando i vari item in base alla coerenza dimostrata da essi in entrambe le somministrazioni, si può notare come quelli che in ambedue gli anni si raggruppano sotto il primo fattore $(15,16,17,18,25,28,29,30)$ esprimono contenuti di tipo «interattivo», o comunque legati all'atteggiamento dell'insegnante nei confronti degli allievi:

15. L'insegnante $\mathrm{mi} / \mathrm{ci}$ ascolta.

16. Con l'insegnante si va d'accordo durante la lezione

17. Con l'insegnante si va d'accordo fuori dalla lezione

18. L'insegnante incoraggia la partecipazione

25. Quando faccio domande l'insegnante mi risponde in modo chiaro e soddisfacente

28. Quando c'è un calo di interesse l'insegnante se ne accorge e cerca di fare qualcosa

29. L'insegnante valorizza la persona dell'apprendista

30. L'insegnante è giusto

Invece, gli item che possono essere ricondotti al secondo fattore in entrambe le somministrazioni $(1,2,3,4,5,8,13,19,21)$ esprimono dei contenuti di tipo più «didattico»:

1. Ho migliorato le mie conoscenze

2. La cultura generale mi ha aiutato a meglio comprendere aspetti importanti per la mia vita 
3. Le correzioni e le valutazioni mi aiutano a capire i miei errori e a migliorare

4. I materiali che ho a disposizione sono chiari

5. I materiali sono utili per imparare

8. Il ritmo d'insegnamento è adeguato

13. Gli argomenti mi vengono proposti in modo chiaro

19. Gli strumenti (computer, lucidi, ecc.) utilizzati dal docente mi aiutano a meglio comprendere la lezione

21. So perché dobbiamo studiare gli argomenti proposti nelle lezioni di CG

Infine, vi è un terzo fattore, rappresentato nelle due somministrazioni dall'item 7, che si riferisce al grado di difficoltà delle attività programmate:

7. Le attività di CG sono impegnative

Gli item così individuati vengono a costituire, rispetto agli originari 31, un "sottoinsieme», che è stato ulteriormente sottoposto ad analisi statistica.

I valori ottenuti relativi al test $\alpha$ di Cronbach sono stati di 0.87 per il 2004 e di 0.91 per il 2007. Vi è quindi una leggera diminuzione, ma il valore si mantiene comunque elevato. Passando alla varianza spiegata, otteniamo i seguenti valori:

Tableau 4: peso e varianza spiegata dai fattori che mantengono una buona coerenza tra 2004 e 2007

\begin{tabular}{|c|c|c|c|}
\hline \multicolumn{2}{|c|}{ Varianza spiegata 2004} & \multicolumn{2}{c|}{ Varianza spiegata 2007 } \\
\hline \% di varianza & \% cumulata & \% di varianza & \% cumulata \\
\hline 23.724 & 23.724 & 27.690 & 27.690 \\
\hline 19.603 & 43.327 & 20.810 & 48.500 \\
\hline 8.593 & 51.920 & 7.018 & 55.518 \\
\hline
\end{tabular}

Si registra quindi un incremento della varianza spiegata, che passa dal $43 \%$ al $52 \%$ per il 2004 , e dal $49 \%$ al $56 \%$ per il 2007 . Di conseguenza, la modesta diminuzione in termini di affidabilità, segnalata dalla riduzione del valore assoluto al test di Cronbach è ampiamente compensata dall'incremento della varianza spiegata. Analizzando ulteriormente i risultati, appare come alcuni ulteriori item potrebbero essere esclusi, in particolare i n. 2, 8, 19 e 21.

In questo caso, l' $\alpha$ di Cronbach ottiene i seguenti valori: 0.85 (2004) e 0.88 (2007). La varianza spiegata evolve invece in questo modo: 
Tableau 5: peso e varianza spiegata dai fattori che mantengono una buona coerenza tra 2004 e 2007, con ulteriore esclusione dei n. 2, 8, 19 e 21

\begin{tabular}{|c|c|c|c|}
\hline \multicolumn{2}{|l|}{ Varianza spiegata 2004} & \multicolumn{2}{|l|}{ Varianza spiegata 2007 } \\
\hline \% di varianza & \% cumulata & \% di varianza & \% cumulata \\
\hline 29.997 & 29.997 & 33.344 & 33.344 \\
\hline 20.287 & 50.285 & 19.981 & 53.325 \\
\hline 8.285 & 58.569 & 8.646 & 61.971 \\
\hline
\end{tabular}

In effetti, a fronte di una piccola riduzione dell'affidabilità (che rimane comunque anche in questo caso elevata) si ottiene un notevole miglioramento della varianza spiegata, che passa al 59\% per il 2004 e al 62\% per il 2007. La seguente tabella, relativa alla distribuzione dei pesi dei vari item, mostra inoltre come gli item proposti discriminino bene in funzione della loro appartenenza ad un fattore o ad un altro, in entrambe le somministrazioni.

Tableau 6: matrice dei componenti dei tre fattori presi in esame nella sua versione finale

\begin{tabular}{|c|c|c|c|c|c|c|c|}
\hline & \multicolumn{3}{|c|}{ Componenti 2003} & \multicolumn{4}{|c|}{ Componenti 2007} \\
\hline & 1 & 2 & 3 & & 1 & 2 & 3 \\
\hline VAR00016 & .821 & .010 & .023 & VAR00030 & .831 & .230 & .025 \\
\hline VAR00015 & .781 & .138 & -.126 & VAR00017 & .791 & .053 & .038 \\
\hline VAR00017 & .711 & .063 & -.060 & VAR00025 & .767 & .206 & .014 \\
\hline VAR00025 & .670 & .361 & -.080 & VAR00016 & .760 & .267 & -.140 \\
\hline VAR00030 & .642 & .438 & .014 & VAR00015 & .687 & .382 & .020 \\
\hline VAR00029 & .633 & .252 & .272 & VAR00028 & .626 & .271 & .255 \\
\hline VAR00018 & .606 & .272 & .052 & VAR00029 & .614 & .390 & -.012 \\
\hline VAR00028 & .601 & .393 & .061 & VAR00018 & .602 & .359 & .139 \\
\hline VAR00005 & .141 & .787 & .021 & VAR00004 & .201 & .808 & -.101 \\
\hline VAR00004 & .186 & .763 & -.170 & VAR00005 & .220 & .769 & -.015 \\
\hline VAR00003 & .172 & .667 & .166 & VAR00001 & .315 & .591 & .165 \\
\hline VAR00001 & .188 & .588 & .153 & VAR00003 & .244 & .577 & .356 \\
\hline VAR00007 & -.041 & .089 & .943 & VAR00007 & -.018 & .036 & .923 \\
\hline
\end{tabular}

Di conseguenza, possono essere considerati, per una versione allo stesso tempo meno "pesante» e più efficace del questionario, i seguenti item: 
A. Dimensione «interazione insegnante/allievi»

15. L'insegnante $\mathrm{mi} / \mathrm{ci}$ ascolta.

16. Con l'insegnante si va d'accordo durante la lezione

17. Con l'insegnante si va d'accordo fuori dalla lezione

18. L'insegnante incoraggia la partecipazione

25. Quando faccio domande l'insegnante mi risponde in modo chiaro e soddisfacente

28. Quando c'è un calo di interesse l'insegnante se ne accorge e cerca di fare qualcosa

29. L'insegnante valorizza la persona dell'apprendista

30. L’insegnante è giusto

B. Dimensione "efficacia della didattica»

1. Ho migliorato le mie conoscenze

3. Le correzioni e le valutazioni mi aiutano a capire i miei errori e a migliorare

4. I materiali che ho a disposizione sono chiari

5. I materiali sono utili per imparare

Un discorso a parte merita invece il terzo fattore. Nei fatti, la dimensione che esso rappresenta (e che potrebbe essere denominata "difficoltà delle attività proposte») si riduce ad un solo item («Le attività di CG sono impegnative»), il quale, tuttavia, dimostra di essere particolarmente efficace (varianza spiegata $8-9 \%$, con orientamento molto netto nella direzione della dimensione rappresentata, confermato nel corso del tempo). Di conseguenza, al momento delle prossime somministrazioni del questionario, verranno sperimentati altri item per cercare di dare più "consistenza» a questa dimensione e poterla così affiancare alle prime due.

Infine, gli item ottenuti sono stati sottoposti ad un panel di 15 giudici per una validazione di contenuto. In sostanza, si è chiesto a questi ultimi di classificarli in base alle tre dimensioni individuate (A, B, C). La correttezza nella classificazione degli item ha dato i seguenti risultati:

Tableau 7: congruenza del giudizio espresso dai gindici sul contenuto degli item rispetto alle aspettative

\begin{tabular}{|l|l|}
\hline VAR00016 & $100 \%$ \\
\hline VAR00015 & $100 \%$ \\
\hline VAR00017 & $100 \%$ \\
\hline VAR00025 & $100 \%$ \\
\hline VAR00030 & $100 \%$ \\
\hline VAR00029 & $100 \%$ \\
\hline
\end{tabular}




\begin{tabular}{|l|r|}
\hline VAR00018 & $100 \%$ \\
\hline VAR00028 & $100 \%$ \\
\hline VAR00005 & $100 \%$ \\
\hline VAR00004 & $100 \%$ \\
\hline VAR00003 & $87 \%$ \\
\hline VAR00001 & $87 \%$ \\
\hline VAR00007 & $100 \%$ \\
\hline
\end{tabular}

Alla luce dei valori molto elevati riscontrati, si è rinunciato a un'analisi dell'affidabilità, in quanto essa risulta implicata dai risultati ottenuti. Due item hanno sollevato qualche dubbio da parte di due diversi giudici (un item per giudice), ossia il n. 1 («Ho migliorato le mie conoscenze») e il n. 3 («Le correzioni e le valutazioni mi aiutano a capire i miei errori e a migliorare): ad essi è stata riconosciuta solo una parziale affiliazione rispetto al secondo fattore (efficacia della didattica). Tuttavia, alla luce del giudizio espresso dal gruppo dei giudici nel suo insieme, si può parlare di una congruenza elevata rispetto al costrutto ipotizzato.

\section{Conclusion $i$}

Il Questionario per la valutazione della didattica e della relazione con l'insegnante è uno strumento che è stato creato presso la SPAI di Mendrisio nel corso di una serie di azioni volte al miglioramento della qualità dell'offerta formativa in tale istituto scolastico. Esso è stato sviluppato mediante una collaborazione tra vari stakeholders della scuola e uno School Improvement Advisor/researcher (SIA) e si è rivelato particolarmente utile nel condurre gli insegnanti ad una riflessione sulle loro pratiche di insegnamento e su un loro possibile miglioramento, in un'ottica di valutazione formativa.

Già nella sua forma originale, con 31 item, lo strumento ha dato prova di elevata affidabilità, mentre la varianza spiegata esprimeva un valore attorno al $50 \%$. Mediante una procedura di riduzione del numero di item, in base al comportamento dello strumento durante due somministrazioni avvenute nel corso del tempo (2004 e 2007), si è giunti a definire un insieme ideale costituito da 13 item, che, pur mantenendo nella sostanza il livello di affidabilità, ha tuttavia permesso di aumentare in modo significativo la varianza spiegata, che è passata a valori attorno al $60 \%$.

Ai fini di assicurare una validità del costrutto ipotizzato (aspetti didattici e relazionali dell'insegnamento), i dati ottenuti mediante l'analisi delle componenti principali sono stati incrociati con i dati risultanti da una classificazione degli item, espressi da un panel di 15 giudici. Quest'ultima analisi ha messo in luce come il gruppo di esperti abbia classificato gli item proposti in modo estremamente congruente con quanto risultante dall'analisi fattoriale. I due approcci 
hanno quindi messo in luce una notevole coerenza, un fatto che testimonia senza dubbio a favore della validità del costrutto ipotizzato. L'item «Le attività di CG sono impegnative» mette in luce la possibilità di sviluppare, mediante l'introduzione e la sperimentazione di item aggiuntivi, un'ulteriore sottoscala del questionario nelle sue versioni future, relativa alla difficoltà delle attività proposte, la quale potrebbe fornire ulteriori informazioni agli insegnanti.

In conclusione, si può affermare che il Questionario per la valutazione della didattica e della relazione con l'insegnante è un dispositivo utile al miglioramento delle pratiche professionali e relazionali messe in atto dai docenti, il quale mette loro a disposizione informazioni valide e affidabili nell'ambito di due importanti dimensioni dell'insegnamento.

In particolare, tale strumento si rivela particolarmente efficace nell'ambito di processi di riflessione sulla pratica professionale assistiti da consulenti, quali ad esempio il SIA, mediante i quali l'insegnante può mettere in atto degli interventi di miglioramento a livello didattico e relazionale in grado di dare delle risposte puntuali a quanto emerso dal giudizio espresso dagli studenti.

\section{Note}

1 Dispositivo di autovalutazione e autoanalisi del piano quadro: si tratta di un processo di autovalutazione riferito in particolare all'applicazione dei programmi di sede (piani quadro) nel settore della formazione professionale.

\section{Bibliografia}

Aleamoni, L. (1981). Student ratings of instruction. In J. Millman (Ed.), Handbook of Teacher Evaluation (pp. 110-145). Beverly Hills: Sage.

Angelo, T. \& Cross, P. (1993). Classroom assessment techniques. San Francisco: Jossey-Bass.

Berger, E., Bottani, N., Soussi, A., Ostinelli, G., Gavreau, C. \& Rhyn, H. (2004). De l'émergence du courant School improvement et exemples d'applications. Revue Française de Pédagogie, 148, 119-133.

Bottani, N. \& Cenerini, A. (2003). Una pagella per la scuola. La valutazione tra autonomia e equità. Trento: Erickson.

Castoldi, M. (1999). Sistemi e modelli per l'analisi dell'insegnamento: Origini e svolgimenti attuali. Ricerche pedagogiche, 131, 59-65.

Castoldi, M. (2004). Valutazione dell'insegnamento e sviluppo professionale. In E. Berger $\&$ F. Pesce (Ed.), Verso una scuola che apprende. Strategie innovative per uno sviluppo. Bellinzona: DECS.

Comoglio, M. (2011). Perché valutare l'insegnamento. Dirigenti Scuola - Annuario 2011. Brescia: La Scuola.

Darling-Hammond, L., Wise, A. \& Pease, S. (1983). Teacher evaluation in the organizational context: A review of the literature. Review of Educational Research, 53, (3), 285-328.

De Charms, R. (1968). Personal causation. New York: Academic Press.

Deci, E. \& Ryan, R. (1985). Intrinsic motivation and self-determination in human behavior. New York: Plenum Press.

Ellett, C. \& Teddlie, C. (2007). Teacher evaluation, teacher effectiveness and school effectiveness : Perspectives from the USA. The Journal of Personnel Evaluation in Education, 17, (1), 101-128.

Joint Committee on Standards for Educational Evaluation (JCSEE). (1988). The personnel evaluation standards - How to assess systems for evaluating educators. Newbury Park: Sage. 
Kulik, J. (2001). Student ratings: Validity, utility and controversy. In M. Theall, P. Abrami \& L. Mets (Ed.), The student ratings debate: Are they valid? How can we best use them? (pp. 9-25). San Francisco: Jossey-Bass.

Lewis, K. (2001). Using midsemester student feedback and responding to it. In K. Lewis (Ed.), Techniques and strategies for interpreting student evaluations (pp. 33-44). San Francisco: Jossey-Bass.

Marsh, H. (1987). Students' evaluations of university teaching: Research findings, methodological issues, and directions for future research. International Journal of Educational Research, 11, 253-388.

McKeachie, W. (1987). Can evaluating instruction improve teaching? In L. Aleamoni (Ed.), Techniques for evaluating and improving instruction (pp. 3-8). San Francisco: Jossey-Bass.

OCSE/CERI. (1994). Valutare l'insegnamento: Per una scuola che conti. Roma: Armando.

Ostinelli, G. (2005). Motivazione e comportamento. Trento: Erickson.

Ostinelli, G. (2007). Il miglioramento della qualità nella scuola e il ruolo chiave del consulente educativo. Orientamenti Pedagogici, 54, (4), 713-734.

Ostinelli, G. (2008a). L'action du «School Improvement Advisor/researcher (SIA)» dans l'amelioration des systemes scolaires contemporains. Paper presentato al colloque Admée, Ginevra.

Ostinelli, G. (2008b). The school improvement advisor/researcher (SIA): Helping the individual school in the foundation and organization of the self-managed improvement. Paper presentato al congresso ECER, Gothenburg.

Ostinelli, G. (2009). Teacher education in Italy, Germany, England, Sweden and Finland. European Journal of Education, 44, (2), 291-308.

Peterson, K. (2000a). Teacher evaluation: A comprehensive guide to new directions and practices. Thousand Oaks: Corwin Press.

Peterson, K. (2000b). Student surveys for school teacher evaluation. Journal of Personnel Evaluation in Education, 14, (2), 135-153.

Ribolzi, L. (2011). Valutare gli insegnanti. Vale la pena? Dirigenti Scuola - Annuario 2011. Brescia: La Scuola.

Schön, D. (1987). Educating the reflective practitioner. San Francisco: Jossey-Bass.

Scriven, M. (1987). Summative teacher evaluation. In J. Millman (Ed.), Handbook of teacher evaluation (pp. 244-271). Beverly Hills: Sage.

Sergiovanni, T. \& Starratt, R. (2003). Valutare l'insegnamento. Roma: LAS.

Stevens, J. (1987). Using student ratings to improve instruction. In L. Aleamoni (Ed.), Techniques for evaluating and improving education (pp. 33-38). San Francisco: Jossey-Bass.

Stumpf, S. \& Rindova, V. (1997). Assessment and evaluation techniques. In J. Bess (Ed.), Teaching well and liking it (pp. 268-284). Baltimore: John Hopkins University Press.

Svinicki, M. (2001). Encouraging your students to give feedback. In K. Lewis (Ed.), Techniques and strategies for interpreting student evaluations (pp. 17-24). San Francisco: Jossey-Bass.

Theall, M. \& Franklin, J. (2001). Looking for bias in all the wrong places: A search for truth or a witch hunt in student ratings of instruction. In M. Theall, P. Abrami, L. Mets (Ed.), The student ratings debate: Are they valid? How can we best use them? (pp. 45-58). San Francisco: Jossey-Bass.

Vertecchi, B. (2003). Manuale della valutazione. Milano: Franco Angeli.

Wiggins, G. \& Mc Tighe, J. (1998). Understanding by design. Study Guide. Alexandria: ASCD.

Wilson, R. (1987). Towards excellence in teaching. In L. Aleamoni (Ed.), Techniques for evaluating and improving instruction (pp. 9-24). San Francisco: Jossey-Bass.

Parole chiave: Valutazione dell'insegnamento, miglioramento dell'insegnamento, riflessione sulle pratiche professionali, relazione insegnanti/allievi, School Improvement Advisor/researcher 


\section{Konzeption und Realisierung des Fragebogens zur Beurteilung des Unterrichts und der Beziehung zur Lehrperson}

\section{Zusammenfassung}

Die Beurteilung von Lehrpersonen ist immer noch ein wichtiges und gleichzeitig diffiziles Thema. Obwohl Lehrpersonen kontinuierlich Schülerinnen und Schüler bewerten, sind sie gegenüber der Beurteilung ihres eigenen Unterrichts kritisch eingestellt. Von daher stellt sich die Frage, wie die Beurteilung des Unterrichts unter Berücksichtigung des Respektierens von Professionalität und Persönlichkeit der Lehrpersonen zu erfolgen hat.

Dazu können Prozesse der Schulentwicklung genutzt werden, wie das Beispiel der Scuola Professionale e Artigianale (SPAI) von Mendrisio (Schweiz) zeigt. Im Rahmen eines Bundes-Teilprojekts im Bereich Selbstevaluation von Schulen (DAAP) entwickelte eine Arbeitsgruppe, die repräsentativ für die Schule zusammengesetzt war und aus Lehrpersonen, Schülerinnen und Schülern, Personen aus der Verwaltung etc. bestand, einen Fragebogen für die Beurteilung des Unterrichts und der Lehrer-Schüler-Beziehung. Dabei wurde die Arbeitsgruppe durch eine Fachperson für Schulentwicklung begleitet. Dieser Artikel beschreibt die einzelnen Arbeitsschritte der Instrumentenentwicklung und die Massnahmen, welche getroffen wurden, um die Gültigkeit und Zuverlässigkeit im Hinblick auf eine umfangreichere Nutzung des Instruments durch italienisch sprechende Lehrpersonen zu erhöhen.

Schlagworte: Beurteilung von Lehrpersonen, Verbesserung der Unterrichtsqualität, Reflexion über Lehrmethoden, Beziehung zwischen Lehrperson und Schülerin/Schüler, School Improvement Advisor/researcher

\section{Conception et réalisation du Questionnaire pour l'évaluation de l'enseignement et de la relation avec l'enseignant}

\section{Résumé}

L'évaluation de l'enseignement a toujours été un thème important bien que difficile à réaliser. Alors même que l'enseignant évalue en permanence, on constate que, dans la plupart des cas, il n'aime pas être évalué. Comment dès lors introduire des formes d'évaluation qui soient à la fois rigoureuses, respectueuses de la professionnalité et de la personnalité des enseignants?

La mise en place d'un environnement orienté vers l'amélioration des institutions scolaires peut être une occasion favorable au développement de telles formes d'évaluation. C'est, en bref, ce qui s'est passé à la Scuola Professionale e Artigianale (SPAI) de Mendrisio au cours de ces dernières années. Concrètement, dans le cadre d'un projet fédéral consacré au développement de formes 
d'autoévaluation à l'école (DAAP), un groupe de travail représentatif des différents acteurs de l'école (enseignants, élèves, direction, etc.) a développé, avec le soutien d'un School Improvement Advisor / researcher (SIA), un questionnaire pour l'évaluation de l'enseignement et de la relation entre enseignant et élèves. Cet article décrit d'une part les étapes qui ont conduit à la création de cet instrument et d'autre part les processus qui ont permis d'en améliorer la validité et la fiabilité, dans le but d'une diffusion à plus large échelle auprès des enseignants tessinois et de langue maternelle italienne.

Mots-clés: Évaluation de l'enseignement, amélioration de l'enseignement, réflexion sur les pratiques professionnelles, relation entre enseignants et élèves, School Improvement Advisor/researcher

\section{Design and realization of the Questionnaire for the evaluation of teaching and the relationship with the teacher}

\section{Summary}

The evaluation of teaching has always been a theme at the same time important and difficult to achieve. While a teacher continuously assesses other individuals during her/his career, it is rather common that s/he doesn't like to be evaluated. Is it really possible to introduce forms of assessment able to be at the same time rigorous and respectful of both professionalism and personality of teachers?

The development of an improvement-oriented environment inside individual schools may be a positive opportunity to give an answer to this question, and, in short, that's exactly what happened at the Scuola Professionale e Artigianale (SPAI) of Mendrisio (Switzerland) during the last years. In fact, as part of a federal project aimed at developing experiences in the domain of school self-evaluation (DAAP) a workgroup, representative of all school's stakeholders (teachers, students, management, etc.), developed, with the consultancy of a School Improvement Advisor/researcher (SIA), a questionnaire aimed at the assessment of both teaching and interaction between teacher and students. This article describes the steps that led to the creation of this instrument and the processes targeted at the improvement of its validity and reliability, with the scope of its use on a larger scale by Italian-speaker teachers.

Keywords: Teaching assessment, teaching improvement, reflection on teaching practices, teacher-students relationship, School Improvement Advisor/ researcher 